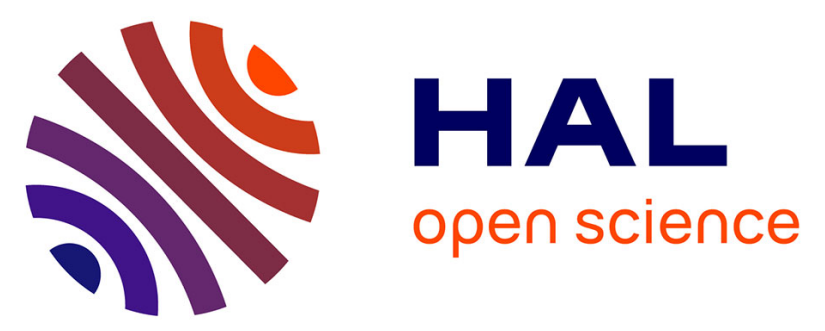

\title{
Driving behavior correction in human-vehicle interaction based on Dynamic Window Approach
}

Yue Kang, Danilo Alves de Lima, Alessandro Corrêa Victorino

\section{To cite this version:}

Yue Kang, Danilo Alves de Lima, Alessandro Corrêa Victorino. Driving behavior correction in humanvehicle interaction based on Dynamic Window Approach. IEEE International Conference on Cyber Technology in Automation, Control and Intelligent Systems (CYBER 2014), Jun 2014, Hong Kong, China. pp.485 - 490, 10.1109/CYBER.2014.6917512 . hal-01120339

\section{HAL Id: hal-01120339 \\ https://hal.science/hal-01120339}

Submitted on 25 Feb 2015

HAL is a multi-disciplinary open access archive for the deposit and dissemination of scientific research documents, whether they are published or not. The documents may come from teaching and research institutions in France or abroad, or from public or private research centers.
L'archive ouverte pluridisciplinaire HAL, est destinée au dépôt et à la diffusion de documents scientifiques de niveau recherche, publiés ou non, émanant des établissements d'enseignement et de recherche français ou étrangers, des laboratoires publics ou privés. 


\section{Driving Behavior Correction in Human-Vehicle Interaction based on Dynamic Window Approach}

\author{
Yue Kang, Danilo Alves de Lima, Alessandro Corrêa Victorino
}

\begin{abstract}
This paper presents the approach of an applicable safety driving methodology for human drivers with focus on human-vehicle interaction. The approach is based on Dynamic Window Approach (DWA) in co-operation with perception of the obstacles. The human driving behaviors are modelled for the design of controller, refined by referential paths using evasive trajectory model, where linear and angular velocities are limited and corrected by DWA which performed as an obstacle avoidance strategy. Results of trajectory following and obstacle avoidance are compared with the Visual Servoing (VS) controller as a corresponding approach of autonomous control pattern.
\end{abstract}

Keywords-Human-Vehicle Interaction, Human Driving Behavior, Driving Safety, Dynamic Window Approach

\section{INTRODUCTION}

Human-Vehicle interaction has been of major interest since the very beginning of the intelligent vehicle history. The interaction, initially limited to the primary commands as steering wheel, accelerator and brake, has evolved to a general level where drivers have access to the knowledge of the vehicle state [1]. For instance, in the project CeMVocAS [2], a first attempt to examine the driver workload while coping with the oncoming phone calls and to apply this concept to an on-vehicle speech recognition system was made. In a more recent approach, the project AIDE [3] involved a real-time awareness system of traffic and environmental conditions. These conditions build up a provision of the information to the driver as simultaneous messages and warnings with the Interaction and Communication Assistant (ICA). During the progress of development, the main responsibility of the human-vehicle interaction remains steady: to enhance the driving safety.

Developing effective active safety devices has gained emphasis within the last decades for accident prevention and harm reduction [4]. A large number of road accidents occur on the main and secondary roads each year, and driver error is one of the reasons with evident importance [5]. A growing number of applicable approaches has been brought out in the progress of various modelings of driving behavior. For instance, a similar system is proposed in [6] which takes into account vehicle dynamics and driver behavior as coefficient that express how much acceleration the driver could accept. Another approach of software-based collision avoidance system was implemented to a dedicated short-range vehicle-to-vehicle communication for better mitigating accident collisions [7]. More recently in [8] a safety-based approaching behavioural model was proposed for the driver's car-following behavior with various driving characteristics, deducted from an integrated driving behavior modeling framework in previous works [9] [10].

However, these achievements focusing on modeling the characteristics and behavior of intelligent vehicles rarely employ the driver's control instructions to the vehicles, and human effect during the driving has failed to be taken into sufficient consideration while human drivers' repulsive controlling characteristics are still major causes of road crashes [11] [12]. As a complement of Advanced Driver Assistance Systems (ADASs) with focus on the human-vehicle interaction, especially the judgement and correction of human drivers'

The authors are with Heudiasyc UMR CNRS 7253 Université de Technologie de Compiègne. Yue Kang holds a Ph.D scholarship from Chinese Scolarship Council and Picardi region in France. Contact authors yue.kang@hds. utc. fr behavior, our approach aims at taking the decision of human drivers into consideration by modeling the driving behaviors, especially the faults and potential dangers. During the driving, the autonomous system interacts with the human driver by detecting these driving behaviors and providing reactions of correction.

Modeling the decision of human drivers preserves its significance as the foundation for the approaches of the judgement and correction of human drivers' behavior in human-vehicle interactions. In [13] a stochastic car-following driver behavior modeling is proposed with operations of the gas and brake pedals in response to the stimuli of vehicle velocity and following distance. a more detailed model of driving behavior characteristics analysis is illustrated in [14] with a weight determination based on influences of various driving behaviors on conflict.

The objective of our approach is to simulate and correct the human drivers' decision faults, which is accomplished by applying the Dynamic Window Approach (DWA) [15]. The human decision faults are modelled as predefined trajectory paths where potential collisions and crashes occur [16]. This approach is emerged from the approach of our parallel work where the DWA method was applied with an image-based Visual Servoing (VS) controller to perform the autonomous road lane following task while avoiding obstacles. In the current approach we focus on the correction of the human decision faults in the same driving environment of the autonomous VSDWA cooperative control pattern, and a comparaison of performance between the two controllers.

In order to present this methodology, this article is organized as follows: in Section II, we present the robot model and the simulation environment; in Section III, we illustrate our design of control pattern in the perspective of human-vehicle interaction with a short introduction of DWA-VS controller used for the comparaison, and the evasive trajectory as the reference for the design of HDB controller; finally experimental results with performance analysis are presented in section IV.

\section{General Definitions}

The robot in this work is considered to move in a planar workspace, similar to the one described in [17], where the road lane center defines a path once differentiable in $\mathbb{R}^{2}$. The robot follows the path according to the kinematic model of a front wheel car [18] as:

$$
\left[\begin{array}{c}
\dot{x_{r}} \\
\dot{y_{r}} \\
\dot{\theta} \\
\dot{\phi}
\end{array}\right]=\left[\begin{array}{c}
\cos \theta \cos \phi \\
\sin \theta \cos \phi \\
\sin \phi / l \\
0
\end{array}\right] v_{1}+\left[\begin{array}{l}
0 \\
0 \\
0 \\
1
\end{array}\right] v_{2},
$$

where the vehicle configuration is given by $q=\left[\begin{array}{lll}x_{r} & y_{r} & \theta\end{array}\right]^{T}$, with the position $\left(x_{r}, y_{r}\right)$ and orientation $(\theta)$ of the car's reference frame $\{\mathcal{R}\}$ in relation to a static world reference frame $\{\mathcal{O}\}$, and $\phi$ is the average steering angle of each front wheel by the Ackerman's approximation. The orientation and steering angles $(\theta$ and $\phi)$ are positive counter-clockwise, with $\theta \in]-\pi, \pi]$ and $\phi \in\left[-\phi_{\max }, \phi_{\max }\right]$. The variables are illustrated in the Figure 1.

The control input for the vehicle of the model (1) is $u=\left[\begin{array}{ll}v_{1} & v_{2}\end{array}\right]^{T}$, which consists of respectively the linear velocity $v_{1}$ and the steering velocity $v_{2}$ of the front wheels. With the relationship between linear velocity $v$ and front wheels velocity $v_{1}$ as $v=v_{1} \cos (\phi)$, as well as the angular velocity $\dot{\theta}=v_{1} \cos (\phi) / r_{1}=\omega$ and the steering angle, it is possible to choose the robot control input as $u_{r}=[v \omega]^{T}$.

For the presentation of Visual Servoing (VS) control used for further comparaisons in the Section III and IV, the Figure 1 also represents the camera frame $\{\mathcal{C}\}$ with optical center position in $\left(x_{c}, y_{c}, z_{c}\right)=\left(t_{x}, t_{y}, t_{z}\right)$ in the robot frame and a constant tilt offset 


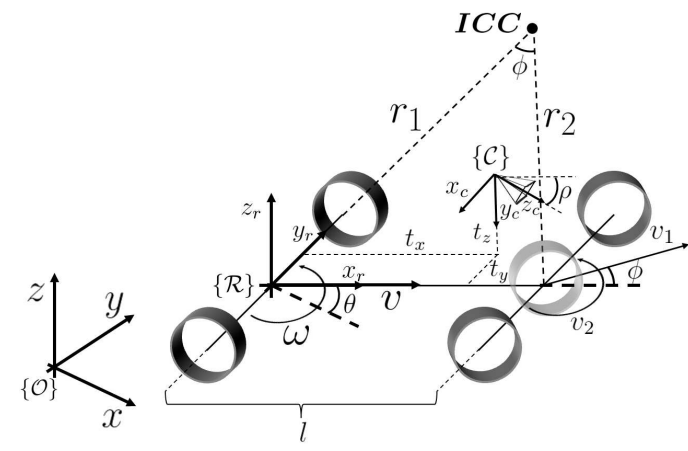

Fig. 1. Cinematic model diagram for a front wheel car-like robot. In this model the vehicle reference frame $\mathcal{R}$ performs circular trajectories related to the instantaneous center of curvature (ICC). The pinhole camera frame is also represented in $\mathcal{C}$.

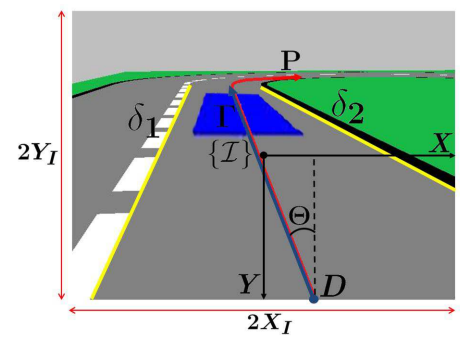

Fig. 2. Image frame $\{\mathcal{I}\}$ with the road lane center projection $P$ (in red) related to the boundaries $\delta_{1}$ and $\delta_{2}$ (in yellow), its tangent $\Gamma$ (in dark blue) at the point $D$ and the angle offset $\Theta$ of $\Gamma$ to the axis $-Y$.

$0<\rho<\frac{\pi}{2}$ related to the $x_{r}$ axis. The camera's image frame $\{\mathcal{I}\}$ is represented in the Figure 2, with defined size as $\left(2 X_{I}, 2 Y_{I}\right)$.

The obstacles on the road are represented as blocks with similar size to our vehicle, as in the Figure 3. An example from the camera point of view is also represented in the Figure 2. To simplify the problem, we assume that the obstacles are in two dimensions, rectangleshaped and can be detected by exteroceptive sensors, for instance, LIDAR. This assumption helps reduce the difficulty of establishing the simulating and experimental environment while maintain the feasibility and reliability of the obstacle avoidance required by the Dynamic Window Approach (DWA).

\section{HUMAN-VEHICLE INTERACTIONS}

Recalling the principal aim of our work, we propose an autonomous correction mechanisme to the driving behaviors based on the Dynamic Window Approach (DWA). The interaction between human drivers and the vehicle, which consists of actions taken by human drivers during the actual driving process, is represented by a predefined referential trajectory with a set of the vehicle configuration $q=$ $\left[\begin{array}{llll}x_{r} & y_{r} & \theta & \phi\end{array}\right]^{T}$ defined in the Section II. This interaction replaces

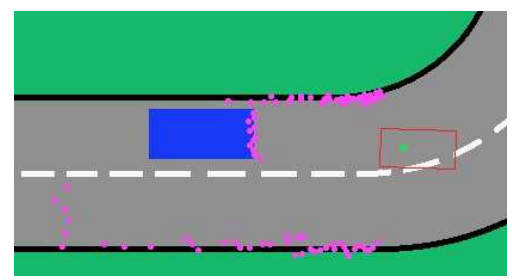

Fig. 3. Obstacles represented in world frame $\{\mathcal{W}\}$ (in blue), with detection of obstacles and also navigable zone boundaries (in pink). the VS control in VS+DWA pattern, and is revised and optimized by the application of the new control input $u=\left[\begin{array}{ll}v_{1} & v_{2}\end{array}\right]^{T}$ calculated by DWA.

\section{A. Dynamic Window Approach}

The Dynamic Window Approach is a reactive obstacle avoidance technique proposed originally by [15], with a modification for carlike robots presented in [19], This approach optimize an objective function (2) in order to select the best control input regarding the desired configuration to the robot. The objective fonction is constituted with three weighted components: the goal position (heading), the obstacle distance (dist) and the final linear velocity (velocity), represented as:

$$
\begin{aligned}
D W A(v, \omega)= & \alpha \cdot \operatorname{heading}(v, \omega)+\beta \cdot \operatorname{dist}(v, \omega) \\
& +\gamma \cdot \operatorname{velocity}(v, \omega) .
\end{aligned}
$$

Initially, for the actual velocity of the robot $\left(v_{a}, \omega_{a}\right)$, the dynamic window $V_{d}$ is defined for all reachable velocities in a time interval $\triangle t$ as:

$$
\begin{aligned}
V_{d}=\{(v, \omega) \mid v & \in\left[v_{a}-\dot{v} \triangle t, v_{a}+\dot{v} \triangle t\right], \\
\omega & \left.\in\left[\omega_{a}-\dot{\omega} \triangle t, \omega_{a}+\dot{\omega} \triangle t\right]\right\}
\end{aligned}
$$

where $\dot{v}$ and $\dot{\omega}$ are respectively the linear and angular acceleration of the robot.

Following, each reachable velocity must be classified as admissible or not depending on the obstacle distance (dist) and the robot maximum breaking accelerations $\left(\dot{v}_{b}, \dot{\omega}_{b}\right)$. The velocity is guaranteed as admissible if the distance to the obstacle in a circular trajectory exceeds the distance required to stop safely the vehicle. The collision detection algorithm implemented in the function $\operatorname{dist}(v, \omega)$ is the one proposed by [20] for polygonal robots, which defines the set of admissible velocities:

$$
\begin{aligned}
V_{a}=\{(v, \omega) \mid v & \leq \sqrt{2 \cdot \operatorname{dist}(v, \omega) \cdot \dot{v}_{b}}, \\
\omega & \left.\leq \sqrt{2 \cdot \operatorname{dist}(v, \omega) \cdot \dot{\omega}_{b}}\right\} .
\end{aligned}
$$

Finally, $V_{s}$ is defined to satisfy the maximum acceleration constraints $\dot{v}_{\max }$ and $\dot{\omega}_{\max }$. The dynamic window search space, which considers the actual speed of the vehicle, its accelerations, the obstacles in the workspace, and also the physical limits of the vehicle is consequently generated as:

$$
V_{D W}=V_{d} \cap V_{a} \cap V_{s} .
$$

\section{B. Design of Control Pattern}

According to the control input for the vehicle defined in the Section II as $u=\left[\begin{array}{ll}v_{1} & v_{2}\end{array}\right]^{T}$, we simplify the human control pattern. In an ideal driving environment where no obstacles are involved, the obstacle distance (dist) in the objective function is omitted. Naturally human driver conducts the vehicle in the center of the road lanes, with which the goal position (heading) in the objective function 2 is determined. The linear and the angular speeds depend on the maximum velocity constrain of the vehicle, the legal speed limitions of the road and the road conditions (turnings, intersections, etc.). In a simplified experimental circle track, the linear velocity $v_{1}$ and the steering velocity $v_{2}$ are constrained respecting these elements.

In general, human drivers avoid the collision with two principal reactions: decelerating and turning. Without any auxiliary perception of the environment, these reactions depend only on the individual judgement of the human drivers [21]. Consequently, the DWA guarantees a limitation of the input velocities with the help of the obstacle avoidance and the implement of the obstacle distance (dist). Nonetheless, apart form the obstacle avoidance, the essential aim of 
human drivers is still guiding the vehicle in the center of the road, and the the goal position (heading) remains unchanged.

As a contrast to the human control pattern, we apply the Visual Servoing (VS) strategy carried out by Cherubini et al [17], in which two non-linear feedback controllers are designed for the propose of following a line path projected in the camera image. With the absence of the capacity to guarantee the safety of driving, a correction by an obstacle avoidance technique, in this case the DWA, is consequently necessary. Omitting the detail of visual perception and projection, the design of the feedback control of VS is represented as:

$$
\omega=-B_{c}^{+}\left(\lambda e+A_{c} v_{d}\right),
$$

where $\omega$ is the feedback gain of the controller, $e$ is the state error calculated according to the visual characteristics from the projected image, $A_{c}$ and $B_{c}$ are matrixes from the kinematic model (1) transformed to the image frame, and $v_{d}$ is the desired linear velocity of the tracking control. For more details of the implementation and stability analysis see [22].

To imitate the behavior of human driving, we propose an ordinary but practicable design of feedback control according to the Euclidean distance between the position $\left(x_{r}, y_{r}\right)$ of the vehicle and a predefined referential trajectory path which represents the desired driving route. This design of feedback control, namely human driving behavior (HDB) controller is represented as:

$$
\omega=\frac{v_{d}}{v_{l}} \sqrt{\left(x_{r}-x_{p}\right)^{2}+\left(y_{r}-y_{p}\right)^{2}}+\beta,
$$

where $\omega$ is the feedback gain of the controller, $v_{d}$ is the desired linear velocity of the tracking control, $v_{l}$ is the velocity limitation of the vehicle, $\left(x_{r}, y_{r}\right)$ are coordinates of the position of the vehicle, $\left(x_{p}, y_{p}\right)$ are coordinates of the corresponding point on the referential path, and $\beta$ is an additive white Gaussian noise (AWGN) which represents inaccuracy of human drivers' control. Noted that this controller involves no angular information. This omission is acceptable in the circumstance where human drivers are rarely aware of the precise drift angle of the vehicle [23].

\section{Referential Path Modeling}

For the requirement of predefinition of the referential path in the design of HDB controller, a mathematical model of driving trajectory is essential. From the approach of vehicle trajectory prediction [11] we are confirmed that, in an appropriately simplified driving circumstance, modeling the human drivers' behaviors with an ensemble of data, including the vehicle position coordinates which consist of a discrete path, and the linear and angular velocities representing the decision of acceleration, brake and steering, is practical and adequate. In an obstacle-free circumstance this path is located in the middle of the drivable road, in the form of segments in a straight line or an arc, depending on the road driving condition characteristics. With obstacles involved, this geometrical layout of driving path is inadequate. In our approach, an evasive trajectory [12] is applied in the task of obstacle avoidance. This trajectory is described by a sigmoid curve in the form as:

$$
y(x)=\frac{y_{m}}{1+e^{-a(x-c)}},
$$

where $y_{m}$ is the maximum $y$-value, $a$ defines the slope at $x=c$ of the sigmoid curve and $c$ defines the position of the inflection point of the curve, therefore the total length as $s=2 c$. The curve of the trajectory along with its parameters is presented in the Figure 4.

Noted that the definition of the evasive trajectory is not a function of time (i.e. an abuse of the naming "trajectory"), we utilize henceforth this model as a segment of a referential path. For the determination

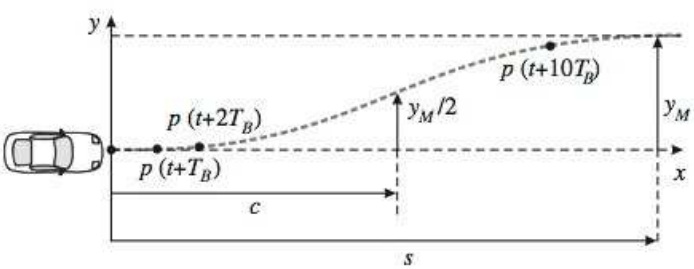

Fig. 4. Evasive trajectory for obstacle avoidance.

of the referential path in the task of obstacle avoidance for HDB controller, we manually revise the initial path, which is obtained in the obstacle-free circumstance, at the segments where obstacles occupy the initial path, as presented in the Figure 5.

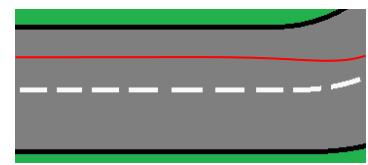

(a) Initial referential path.

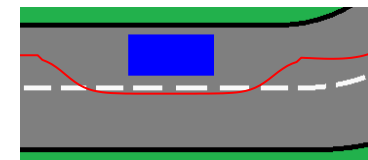

(b) Revised referential path with obstacles.
Fig. 5. Referential path revision using evasive trajectory.

\section{EXPERIMENTAL RESUlTS}

\section{A. Validation}

Our first experiment aims at validating the design of imitational feedback controlling mechanism of the HDB controller that mimics the driving behaviors of humankind. The vehicle is set in a closedloop athletic-track-shaped environment as workspace without obstacles, in which a referential clockwise path in the middle of the rightside road is predefined. The vehicle succeeded the path following task in accordance with the HDB controller applied to the kinematic model (1), as represented in the Figure 6.

\begin{tabular}{r|c}
\hline Direction & Max Error $(\mathrm{m})$ \\
\hline \hline X Axis & 2.58 \\
\hline Y Axis & 1.83 \\
\hline \hline Euclidien Distance & 2.70 \\
\hline \hline
\end{tabular}

TABLE I. MAX ERRORS OF THE DRIVING ROUTE.

The results in the Figure 6(a) demonstrated the driving route by HDB controller with an ideal referential path, while the Figure 6(b) and the Table I illustrated the divergence of the coordinates of the actual driving route in comparison with the reference. While the overall route converged to the referential path, the largest deviation of the driving route occurred when the vehicle left the curve and entered the straight road. This phenomenon is due to the absence of angular information in the design of the HDB controller, which causes time delay of establishing the steady angular state. It also reflects the common failure of the judgement by the human drivers as incorrect estimation of the actual steering angle of the vehicle.

\section{B. Comparison with VS}

For the purpose of proving the feasibility and reliability of our methodology in the sense of safety driving, we set up obstacles showed in the Figure 3 in the simulation environment, and apply DWA method to both HDB and VS controllers. The results are presented in the Figure 7. 


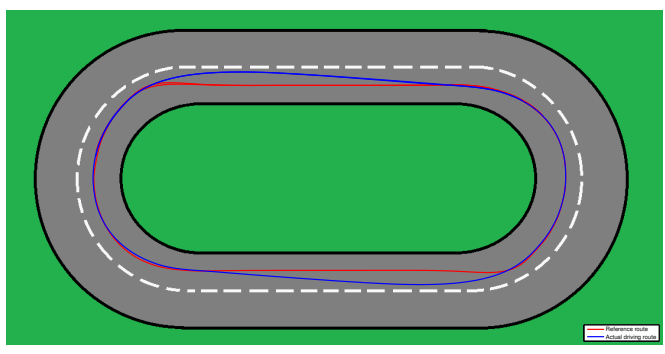

(a) Reference path (in red, hereinafter) and actual driving route by HDB controller (in blue, hereinafter).

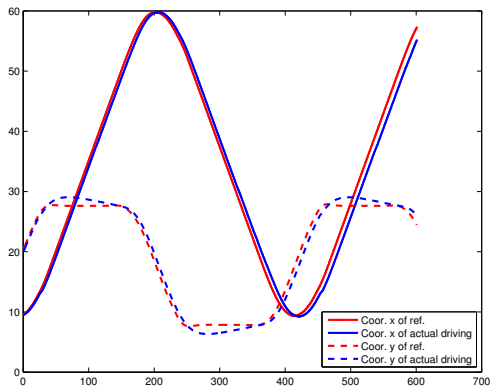

(b) Contrast of coordinates $\mathrm{X}$ and $\mathrm{Y}$ of the two routes.

Fig. 6. Validation of HDB controller design.

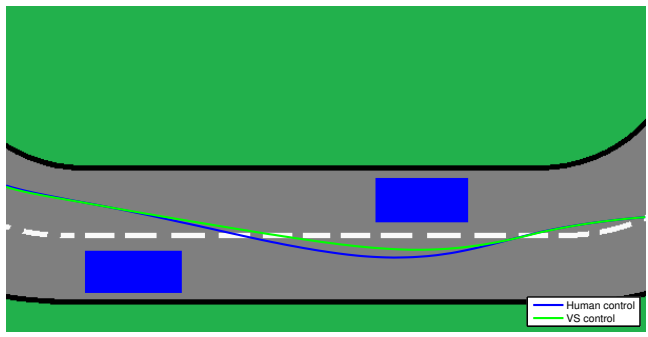

(a) HDB control driving route (in blue, hereinafter) and VS control driving route (in green, hereinafter), both in portion.
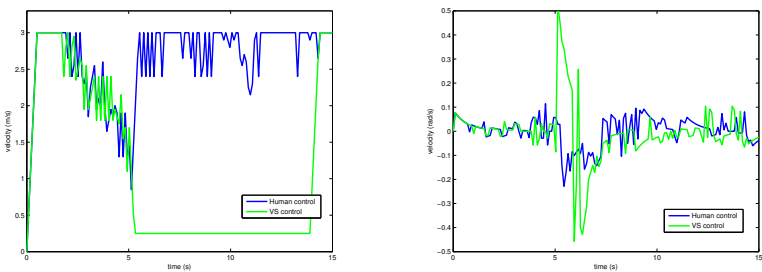

(b) Comparison of linear velocities. (c) Comparison of angular velocities.

Fig. 7. Comparison of performance in obstacle avoidance.
The routing result presented in the Figure 7(a) demonstrated that both controllers succeeded in guiding the robot to avoid obstacles with the application of DWA. Although the difference between the two routes did not prove adequately the advantage of our approach, the Figure 7(b) demonstrated that, after an essential deceleration before engaging the avoidance, the vehicle applied with the HDB controller passed the obstacle while maintaining a relatively high linear velocity. In contrast, with a sharp drop followed by a long period of low-speed forwarding, the robot guided by the VS controller failed to retain the velocity during a passing manoeuvre, which demanded a consequently long passing time and an increase of energy consuming.

\begin{tabular}{r|c|c}
\hline & HDB & VS \\
\hline \hline Respond Moment & $2 \mathrm{~s}$ & $1.8 \mathrm{~s}$ \\
\hline Decelerate Time & $3.4 \mathrm{~s}$ & $3.6 \mathrm{~s}$ \\
\hline Low Speed Passing Time & N/A & $8.5 \mathrm{~s}$ \\
\hline Min Linear Velocity & $0.85 \mathrm{~m} / \mathrm{s}$ & $0.5 \mathrm{~m} / \mathrm{s}$ \\
\hline Max Angular Velocity & $0.23 \mathrm{rad} / \mathrm{s}$ & $0.52 \mathrm{rad} / \mathrm{s}$ \\
\hline \hline
\end{tabular}

TABLE II. COMPARISON OF PERFORMANCE IN OBSTACLE AVOIDANCE.

In addition, the Figure $7(\mathrm{c})$ and the Table II illustrated that HDB controller guaranteed the smoothness of turning procedure. The angular velocity of the robot calculated by HDB controller remained in a suitable interval. As a contrast, with the guidance of the VS controller, the angular velocity rapidly flipped with peak values considerably larger than those calculated by HDB controller. The experimental result proved HDB controller available and feasible with the cooperation of DWA method and a predefined referential path, while reducing the demand of deceleration in comparison with the VS controller.

\section{Danger Behaviors Correction}

In this experimental section, the performance of our methodology is examined in the circumstance where human drivers adopt danger behaviors while driving. For the purpose of representing the danger behaviors, the referential path is adjusted according to the evasive trajectory presented in the Section III-C. Firstly we postpone the turning of the path before bypassing an obstacle to simulate the delayed steering decision of human drivers. Another danger behavior is presented as a wrong turning which leads to a potential thread of collision. Both adjustments of the referential path are presented in the Figure 8(a).

The experimental result of the route, also presented in the Figure $8(a)$, illustrated that the robot successfully avoided the potential danger of collision with the application of HDB controller and VSDWA method. The route taken by the robot applying the HDB controller held a safe distance from the obstacles and simultaneously remained smooth and stable from the perspective of the reference frame. In addition, the Figure 8(b) reclaimed the maintenance of linear velocity during the bypass period, as is mentioned in the Section IV-B. Although a larger interval of angular velocity is observed in the Figure 8(c), in comparison with the result in the Figure 7(c), the peaks during the first period of steering is decreased, and a smooth passing manoeuvre is consequently guaranteed.

In the following experiment, we took a step further to enlarge excessively the hazard of danger driving behavior by canceling the turning of the path before passing an obstacle. In such circumstance the obstacle occupies and blocks completely the referential pas as presented in the Figure 9(a).

The results presented in the Figure 9(a) revealed again a smooth and stable trajectory from the perspective of the reference frame with the manoeuvre of obstacle avoidance. The maintenance of linear velocity 


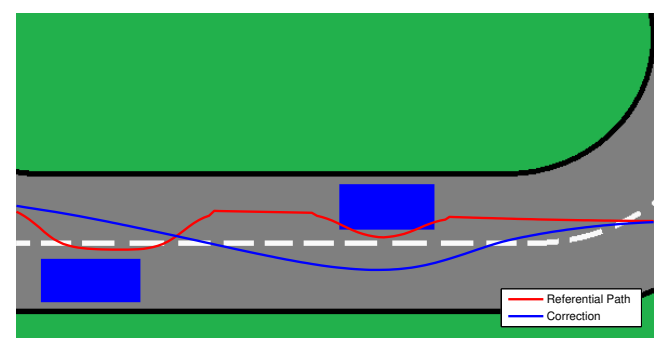

(a) Referential path with danger behaviors (in red) and corrected route by HDB controller (in blue), both in portion.

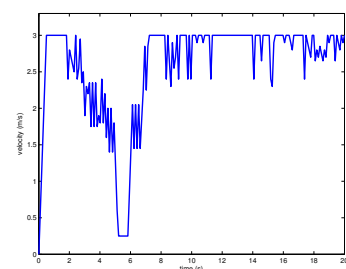

(b) Linear velocity calculated by (c) Angular velocity calculated by HDB controller.

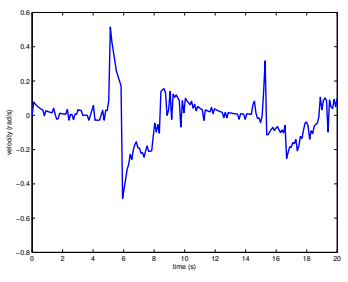

HDB controller.
Fig. 8. Result of danger behaviors correction performed by HDB controller.

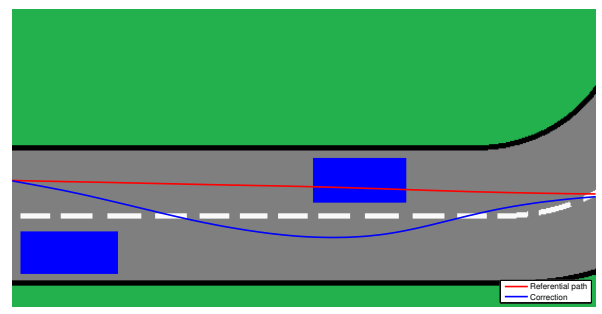

(a) Referential path with inevitable collision (in red) and corrected route by HDB controller (in blue), both in portion.

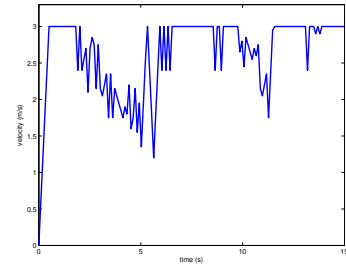

(b) Linear velocity calculated by (c) Angular velocity calculated by HDB controller.

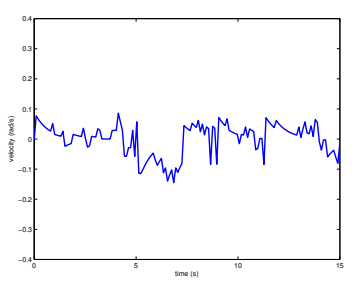

HDB controller.
Fig. 9. Result of danger behaviors correction performed by HDB controller.

during the bypass period is also observed in such circumstance as illustrated in the Figure 9(b). The major concern in this part of experiment lies in the result of the angular velocity showed in the Figure 9(c). In comparison with the results from the previous experiments in the Figure 7(c) and 8(c), the angular velocity by HDB controller in such circumstance revealed a smooth process of steering with minor values of the angular velocity and fewer peaks. Due to the curve characteristics of the referential path, we conclude that the quality of the referential path influences considerably the behavior of HDB controller, thus affecting consequently the outcome of the practical control of the robot.

\section{CONCLUSION}

In this paper we have presented an approach of human-vehicle interaction by modeling the behaviors and decisions of human drivers, which has established the foundation of a simplified control pattern of human drivers' behaviors. With the application of DWA providing the validation of the linear and angular velocities of the vehicle, potential dangerous behaviors of human drivers have been refrained and corrected. Safety of driving in such circumstance has been therefore guaranteed.

In comparison with the experimental results of VS controller, our control pattern with the focus on human-vehicle interaction have proved its superiority in maintaining the smoothness and stability of the obstacle passing manoeuvre. Control strategies determined by human drivers, or in our approach by HDB controller, with both absolute values and smoothness of the control variables being promising, have also been confirmed as practical and applicable on actual intelligent vehicles. The realisation of the experimentations is being executed currently with our vehicle IRIS in the project VERVE $^{1}$.

Our approach has yet called for quite a few further progresses. The most apparent shortcoming of our design is the indispensable predefinition of the referential path or trajectory. A real-time calculation to achieve the referential trajectory from the perspective of human drivers, and a reliable localisation method to guarantee the acquisition of the position of the vehicle, are consequently desired. The characteristics of the referential path has also revealed its significance to the performance of the HDB controller. These current deficiencies will point out the direction of our future research, particularly for our experimental vehicle (Fluence-ZE, a zero emission vehicle from Renault) in order to experimentally validate our HDB method.

\section{REFERENCES}

[1] S. Damiani, E. Deregibus, and L. Andreone, "Driver-vehicle interfaces and interaction: where are they going?" European Transport Research Review, vol. 1, no. 2, pp. 87-96, 2009. [Online]. Available: http://dx.doi.org/10.1007/s12544-009-0009-2

[2] H. Tattegrain-Veste, M. Bruyas, T. Bellet, G. Pachiaudi, J. Forzy, D. Martini, B. Baligand, A. Simoes, J. Carvalhais, P. Lockwood et al., "Evaluation of a voice interface management system," Transportation Research Record: Journal of the Transportation Research Board, vol. 1759, no. 1, pp. 46-51, 2001.

[3] L. Andreone, A. Amditis, E. Deregibus, S. Damiani, D. Morreale, and F. Bellotti, "Beyond context-awareness: driver-vehicle-environment adaptivity. from the comunicar project to the aide concept," in 16th IFAC WORLD CONGRESS, Prague, 2005.

[4] R. Ghandour, A. C. Victorino, A. Charara, and A. Lechner, "Risk indicators evaluation based on anticipated vehicle dynamics parameters," IEEE Intelligent Systems, pp. 68 - 73, 2012.

[5] F. Lotz, "System architectures for automated vehicle guidance concepts," in Automotive Systems Engineering. Springer, 2013, pp. 39-61.

[6] S. Glaser, L. Nouveliere, and B. Lusetti, "Speed limitation based on an advanced curve warning system," in Intelligent Vehicles Symposium, 2007 IEEE. IEEE, 2007, pp. 686-691.

[7] A. Tang and A. Yip, "Collision avoidance timing analysis of dsrc-based vehicles," Accident Analysis \& Prevention, vol. 42, no. 1, pp. 182-195, 2010.

[8] W. Wang, W. Zhang, H. Guo, H. Bubb, and K. Ikeuchi, "A safety-based approaching behavioural model with various driving characteristics," Transportation research part C: emerging technologies, vol. 19, no. 6, pp. 1202-1214, 2011.

${ }^{1}$ The project VERVE stands for Novel Vehicle Dynamics Control Technique for Enhancing Active Safety and Range Extension of Intelligent Electric Vehicles. 
[9] T. Toledo, H. N. Koutsopoulos, and M. Ben-Akiva, "Integrated driving behavior modeling," Transportation Research Part C: Emerging Technologies, vol. 15, no. 2, pp. 96-112, 2007.

[10] - "Estimation of an integrated driving behavior model," Transportation Research Part C: Emerging Technologies, vol. 17, no. 4, pp. 365380, 2009.

[11] A. Houenou, P. Bonnifait, V. Cherfaoui, and W. Yao, "Vehicle trajectory prediction based on motion model and maneuver recognition," in Intelligent Robots and Systems (IROS), 2013 IEEE/RSJ International Conference on, 2013, pp. 4363-4369.

[12] R. Isermann, R. Mannale, and K. Schmitt, "Collision-avoidance systems proreta: Situation analysis and intervention control," Control Engineering Practice, 2012.

[13] P. Angkititrakul, C. Miyajima, and K. Takeda, "Impact of driving context on stochastic driver-behavior model: Quantitative analysis of car following task," in Vehicular Electronics and Safety (ICVES), 2012 IEEE International Conference on, 2012, pp. 163-168.

[14] X. Ge, J. Lu, Q.-J. Xiang, and P.-Y. Wang, "Study on driving behavior and traffic conflict at highway intersection," in Power Electronics and Intelligent Transportation System, 2008. PEITS '08. Workshop on, 2008, pp. 492-495.

[15] D. Fox, W. Burgard, and S. Thrun, "The dynamic window approach to collision avoidance," Robotics \& Automation Magazine, IEEE, vol. 4, no. 1, pp. 23-33, 1997.

[16] D. A. Lima and G. A. Pereira, "Navigation of an autonomous car using vector fields and the dynamic window approach," Journal of Control, Automation and Electrical Systems, pp. 1-11, 2013. [Online]. Available: http://dx.doi.org/10.1007/s40313-013-0006-5

[17] A. Cherubini, F. Chaumette, and G. Oriolo, "Visual servoing for path reaching with nonholonomic robots," Robotica, vol. 29, pp. 1037-1048, 12 2011. [Online]. Available: http://journals.cambridge. org/article_S0263574711000221

[18] A. D. Luca, G. Oriolo, A. De, and C. Samson, Robot Motion Planning and Control. Springer Berlin / Heidelberg, 1998, vol. 229, ch. Feedback Control Of A Nonholonomic Car-Like Robot, pp. 171-253.

[19] K. Rebai, O. Azouaoui, M. Benmami, and A. Larabi, "Car-like robot navigation at high speed," in Proceedings of the IEEE International Conference on Robotics and Biomimetics, 2007, pp. 2053-2057.

[20] K. Arras, J. Persson, N. Tomatis, and R. Siegwart, "Real-time obstacle avoidance for polygonal robots with a reduced dynamic window," in Proceedings of the IEEE International Conference on Robotics and Automation, vol. 3, 2002, pp. 3050-3055.

[21] T. A. Ranney, "Models of driving behavior: a review of their evolution," Accident Analysis \& Prevention, vol. 26, no. 6, pp. 733-750, 1994.

[22] A. Cherubini, F. Chaumette, and G. Oriolo, "An image-based visual servoing scheme for following paths with nonholonomic mobile robots," in Control, Automation, Robotics and Vision, 2008. ICARCV 2008. 10th International Conference on. IEEE, 2008, pp. 108-113.

[23] C. Keller, T. Dang, H. Fritz, A. Joos, C. Rabe, and D. Gavrila, "Active pedestrian safety by automatic braking and evasive steering," Intelligent Transportation Systems, IEEE Transactions on, vol. 12, no. 4, pp. 12921304, 2011. 